Continuum quantum systems as limits of discrete quantum systems. III. Operators

Laurence Barker

Citation: Journal of Mathematical Physics 42, 4653 (2001); doi: 10.1063/1.1398582

View online: http://dx.doi.org/10.1063/1.1398582

View Table of Contents: http://aip.scitation.org/toc/jmp/42/10

Published by the American Institute of Physics

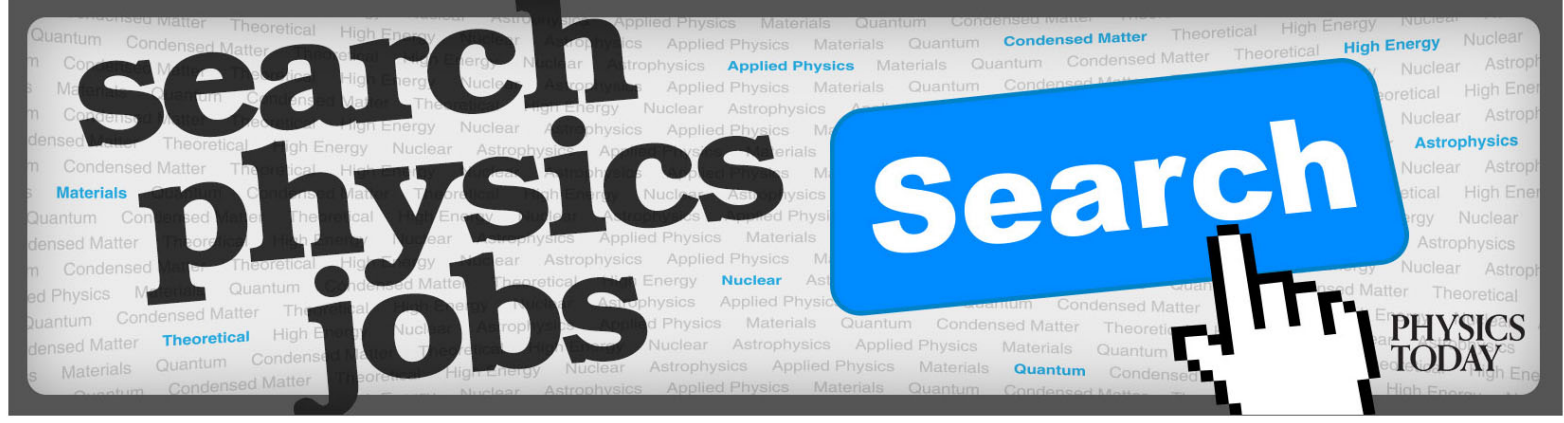




\title{
Continuum quantum systems as limits of discrete quantum systems. III. Operators
}

\author{
Laurence Barker ${ }^{a)}$ \\ Department of Mathematics, Bilkent University, 06533 Bilkent, Ankara, Turkey
}

(Received 29 September 2000; accepted for publication 12 July 2001)

Convergence of a "discrete" operator to a "continuum" operator is defined. As examples, the circular rotor, the one-dimensional box, the harmonic oscillator, and the fractional Fourier transform are realized as limits of finite-dimensional quantum systems. Limits, thus defined, preserve algebraic structure. The results prepare for a sequel in which some affine canonical transforms will be "discretized." (C) 2001 American Institute of Physics. [DOI: 10.1063/1.1398582]

\section{INTRODUCTION}

The continuum fractional Fourier transform of Namias $^{1}$ is the limit of two discrete fractional Fourier transforms, namely, the Kravchuk function FRFT and the Harper function FRFT (see Refs. 2 and 3). Some very straightforward continuum quantum systems, such as the circular rotor, the one-dimensional box and the harmonic oscillator, can easily be realized as limits of equally straightforward finite-dimensional systems whose Hamiltonians are difference operators. For many purposes, the above assertions are clear enough without "limit" being understood to have any abstract meaning; nevertheless, the goal of this article is to assign an appropriate general meaning to "limit," to state the above assertions precisely, and to prove them. It is not that we object to the usual common sense techniques - on the contrary, we shall validate them-but subsequently, in a sequel, ${ }^{4}$ some ideas pioneered by Atakishiyev-Chumakov-Wolf ${ }^{5}$ will be developed: continuum affine canonical transforms and continuum complex-order Fourier transforms will be realized as limits of analogous finite-dimensional transforms. In that application, common sense would not suffice.

Consider a Hilbert space $\mathcal{L}_{\infty}$, and Hilbert spaces $\mathcal{L}_{n}$, where the index $n$ runs over some infinite set of positive integers. In Sec. II, we shall interface $\mathcal{L}_{\infty}$ with the spaces $\mathcal{L}_{n}$, and we shall assign a meaning to equations of the form

$$
\hat{K}_{\infty}=\lim _{n} \hat{K}_{n}
$$

where $\hat{K}_{\infty}$ is a bounded operator on $\mathcal{L}_{\infty}$, and each $\hat{K}_{n}$ is a bounded operator on $\mathcal{L}_{n}$. In Sec. III, we shall assign a meaning to equations of the form

$$
\mathcal{K}_{\infty}=\lim _{n} \mathcal{K}_{n},
$$

where $\mathcal{K}_{\infty}$ and $\mathcal{K}_{n}$ are quantum systems on $\mathcal{L}_{\infty}$ and $\mathcal{L}_{n}$, respectively. Convergence of vectors has already been discussed in two prequels to the present article. The first prequel ${ }^{6}$ explains how $\mathcal{L}_{\infty}$ is to be interfaced with the spaces $\mathcal{L}_{n}$, and gives meaning to equations of the form

$$
\psi_{\infty}=\lim _{n} \psi_{n}
$$

${ }^{a}$ Electronic mail: barker@fen.bilkent.edu.tr. 
where $\psi_{\infty} \in \mathcal{L}_{\infty}$ and $\psi_{n} \in \mathcal{L}_{n}$. Some of the main definitions and results from Ref. 6 are briefly recalled later in Sec. II. The second prequel $^{7}$ shows that widely used limiting techniques are in accordance with the definition of convergence.

With a view to applications, we might think of $\mathcal{L}_{\infty}$ as a "continuum" space, perhaps the Hilbert space formed from the space of square-integrable functions on a differentiable manifold. We might think of each space $\mathcal{L}_{n}$ as a "discrete" space, perhaps a Hilbert space with a coordinate system such that the coefficients of a vector may be interpreted as sample-point values of a function on the manifold. In the case where the manifold is R, Digernes-Varadarájan-Varadhan ${ }^{8}$ established a continuum-discrete correspondence-characterized in terms of limits-by embedding each $\mathcal{L}_{n}$ in $\mathcal{L}_{\infty}$. Our approach is more concerned with preservation of algebraic structure (linearity, inner products, composition, tensor products). We interface $\mathcal{L}_{\infty}$ with the spaces $\mathcal{L}_{n}$ by realizing the sequence $\left(\mathcal{L}_{n}\right)_{n}$ as an inductive resolution of $\mathcal{L}_{\infty}$. The definition of an inductive resolution (recalled in Sec. II) is entirely algebraic, and, by this virtue, it relieves us of any need to assign any abstract meaning to the jargon "continuum" and "discrete." (As every physicist knows, these two terms often refer to different sides of the same coin.)

The preservation of algebraic structure will be crucial in Ref. 4, where we shall be considering some Lie groups with several degrees of freedom. In subsequent work, we shall present a more systematic study of a way in which "continuum" (usually infinite-dimensional) representations of Lie groups may be realized as limits of "discrete" (usually finite-dimensional) representations. (Part of the motive for this is to seek criteria for a system of numerically calculated transforms to respect "continuum" composition laws.) The results we give later, in Sec. III, and the applications we note in Sec. IV, all concern the special case of one-parameter groups. This special case is helpful as a stepping-stone because some of the concerns that arise in the general case reduce to trivialities here.

However, one-parameter systems are of interest in their own right, and can naturally be regarded as quantum dynamical systems, or, to use the language of Parthasarathy, ${ }^{9}$ quantum stochastic processes. (Let us not quibble about the flexible definitions of these terms.) Thus, we are led back to a question addressed by Digernes-Varadarajan-Varadhan. ${ }^{8}$ To what extent are spectra in the "continuum" scenario related to spectra in the "discrete" scenario? This question is explored in Sec. V. The author would like to thank the referee for some useful suggestions concerning Sec. V. Although the material there is still only an initial foray into the matter, it was absent from the previous version of this article.

General motives for a continuum-discrete correspondence-characterized in terms of limits, and preserving algebraic structure-are noted in the prequels, Refs. 6 and 7. Some more extensive references for applications may be found in those two papers. The Gedankenexperiment in Ref. 7, Sec. 2, gives a heuristic introduction to our line of approach.

\section{INDUCTION OF BOUNDED OPERATORS}

By an operator on a Hilbert space $\mathcal{L}$, we mean a linear map $\mathcal{D} \rightarrow \mathcal{L}$, where the domain $\mathcal{D}$ is a dense subspace of $\mathcal{L}$. Every bounded operator on $\mathcal{L}$ extends uniquely to a bounded operator on $\mathcal{L}$ with domain $\mathcal{L}$. Henceforth, all our bounded operators on a Hilbert space $\mathcal{L}$ shall be deemed to have domain $\mathcal{L}$. We write $U(\mathcal{L})$ for the group of unitary operators on $\mathcal{L}$.

We must briefly review some of the definitions and results of Ref. 6. Consider a Hilbert space $\mathcal{L}_{\infty}$, a dense subspace $\mathcal{S}$ of $\mathcal{L}_{\infty}$, an infinite set of positive integers $\mathcal{N}$, Hilbert spaces $\mathcal{L}_{n}$ for each $n \in \mathcal{N}$, and linear maps res ${ }_{n}: \mathcal{S} \rightarrow \mathcal{L}_{n}$. (The results below may easily be extended to the case where $\mathcal{N}$ is any directed set, as in Ref. 6.)

The linear maps res $_{n}$, called the restriction maps, are required to satisfy the reciprocity condition

$$
\langle\phi \mid \chi\rangle=\lim _{n \in \mathcal{N}}\left\langle\operatorname{res}_{n}(\phi) \mid \operatorname{res}_{n}(\chi)\right\rangle
$$


for all $\phi, \chi \in \mathcal{S}$. The sequence $\left(\mathcal{L}_{n}\right)_{n}$, equipped with the sequence $\left(\operatorname{res}_{n}\right)_{n}$, is called an inductive resolution of $\mathcal{L}_{\infty}$.

Given a vector $\psi \in \mathcal{L}_{\infty}$, and vectors $\psi_{n} \in \mathcal{L}_{n}$ for sufficiently large $n \in \mathcal{N}$ (not necessarily for all $n \in \mathcal{N})$, we say that the sequence $\left(\psi_{n}\right)_{n}$ converges to $\psi_{\infty}$ provided the norms $\left\|\psi_{n}\right\|$ are bounded and

$$
\left\langle\phi \mid \psi_{\infty}\right\rangle=\lim _{n \in \mathcal{N}}\left\langle\operatorname{res}_{n}(\phi) \mid \psi_{n}\right\rangle
$$

for all $\phi \in \mathcal{S}$. The Riesz representation theorem guarantees that $\left(\psi_{n}\right)_{n}$ converges to at most one vector in $\mathcal{L}_{\infty}$. When $\left(\psi_{n}\right)_{n}$ converges to $\psi_{\infty}$, we call $\psi_{\infty}$ the limit of $\left(\psi_{n}\right)_{n}$, and we write $\psi_{\infty}$ $=\lim _{n \in \mathcal{N}} \psi_{n}$. Note that $\phi=\lim _{n \in \mathcal{N}} \operatorname{res}_{n}(\phi)$ for all $\phi \in \mathcal{S}$.

Let us recall some results that we shall need from Ref. 6.

Theorem 2.1: (Ref. 6, Theorem 2.4) Any vector $\psi_{\infty} \in \mathcal{L}_{\infty}$ is the limit of some sequence $\left(\psi_{n}\right)_{n}$, and, furthermore, the vectors $\psi_{n} \in \mathcal{L}_{n}$ may be chosen such that $\left\|\psi_{\infty}\right\|=\left\|\psi_{n}\right\|$ for all $n$.

Let $\mathcal{B}_{\infty}=\left\{\beta_{j, \infty}: j \in J_{\infty}\right\}$ be any enumerated orthonormal basis for $\mathcal{L}_{\infty}$. Here, $J_{\infty}=\mathbb{N}$ if $\mathcal{L}_{\infty}$ is infinite-dimensional, while $J_{\infty}=\{0,1, \ldots, d-1\}$ if $\mathcal{L}_{\infty}$ has finite dimension $d$. By Ref. 6, Theorem 3.1, there exist $\mathcal{B}_{n}$, indexed by $n \in \mathcal{N}$, where each $\mathcal{B}_{n}$ is an enumerated orthonormal set $\mathcal{B}_{n}$ $=\left\{\beta_{j, n}: j \in J_{n}\right\}$ in $\mathcal{L}_{n}$, and

$$
\beta_{j, \infty}=\lim _{n \in \mathcal{N}} \beta_{j, n}
$$

for all $j \in J_{\infty}$. Note that, for each basis vector $\beta_{j, \infty}$ in $\mathcal{L}_{\infty}$, a corresponding basis vector $\beta_{j, n}$ in $\mathcal{L}_{n}$ need not exist for all $n$, but the $\beta_{j, n}$ must exist for sufficiently large $n$.

As explained in Ref. 6, Sec. 3, the $\mathcal{B}_{n}$ cannot always be chosen such that each $\mathcal{B}_{n}$ is a basis. (In all our applications in Sec. IV, each of our chosen $\mathcal{B}_{n}$ is a basis. We also mention that, in all these applications, $\mathcal{L}_{\infty}$ is infinite-dimensional, $\mathcal{N}$ is a set of positive integers, and each $\mathcal{L}_{n}$ has finite dimension $n$.) We let $\mathcal{L}_{n}^{\perp}$ denote the subspace of $\mathcal{L}_{n}$ orthogonally complementary to the span of $\mathcal{B}_{n}$. Given a vector $\psi_{\infty} \in \mathcal{L}_{\infty}$, we write

$$
\psi_{\infty}=\sum_{j=0}^{\infty} c_{j, \infty} \beta_{j, \infty}
$$

with the understanding that $c_{j, n}=0$ for all $j \in \mathbb{N}-J_{\infty}$. Given $\psi_{n} \in \mathcal{L}_{n}$, we write

$$
\psi_{n}=\psi_{n}^{\perp}+\sum_{j=0}^{\infty} c_{j, n} \beta_{j, n}
$$

where $\psi_{n}^{\perp} \in \mathcal{L}_{n}^{\perp}$, and $c_{j, n}=0$ for all $j \in \mathbb{N}-J_{n}$. (Of course, if $\mathcal{B}_{n}$ is a basis, then $\psi_{n}^{\perp}=0$.) For later convenience, we define $\beta_{j, \infty}:=0$ when $j \in \mathbb{N}-J_{\infty}$, and $\beta_{j, n}:=0$ when $j \in \mathbb{N}-J_{n}$. Thus $c_{j, \infty}$ $=\left\langle\beta_{j, \infty} \mid \psi_{\infty}\right\rangle$ and $c_{j, n}=\left\langle\beta_{j, n} \mid \psi_{n}\right\rangle$ for all $j \in \mathbb{N}$.

Theorem 2.2: (Ref. 6, Theorem 3.4) Using the notation above, $\psi_{\infty}=\lim _{n \in \mathcal{N}} \psi_{n}$ if and only if the norms $\left\|\psi_{n}\right\|$ are bounded, and $c_{j, \infty}=\lim _{n \in \mathcal{N}} c_{j, n}$ for all $j \in J_{\infty}$.

We can now turn to convergence of operators. Let $\hat{K}_{\infty}$ be a bounded operator on $\mathcal{L}_{\infty}$, and for sufficiently large $n \in \mathcal{N}$, let $\hat{K}_{n}$ be a bounded operator $\mathcal{L}_{n}$. We say that the sequence $\left(\hat{K}_{n}\right)_{n}$ converges to $\hat{K}_{\infty}$ provided the norms $\left\|\hat{K}_{n}\right\|$ are bounded, and for all $\psi_{\infty} \in \mathcal{L}_{\infty}$, and all sequences $\left(\psi_{n}\right)_{n}$ with $\psi_{n} \in \mathcal{L}_{n}$ and $\psi_{\infty}=\lim _{n \in \mathcal{N}}\left(\psi_{n}\right)$, we have

$$
\hat{K}_{\infty} \psi_{\infty}=\lim _{n \in \mathcal{N}}\left(\hat{K}_{n} \psi_{n}\right) .
$$

Theorem 2.1 ensures that the sequence $\left(\hat{K}_{n}\right)_{n}$ converges to at most one bounded operator on $\mathcal{L}_{\infty}$. When $\left(\hat{K}_{n}\right)_{n}$ converges to $\hat{K}_{\infty}$, we call $\hat{K}_{\infty}$ the limit of $\left(\hat{K}_{n}\right)_{n}$, and we write $\hat{K}_{\infty}=\lim _{n \in \mathcal{N}} \hat{K}_{n}$. 
Remark 2.3: Given bounded operators $\hat{K}_{\infty}=\lim _{n \in \mathcal{N}} \hat{K}_{n}$ and $\hat{K}_{\infty}^{\prime}=\lim _{n \in \mathcal{N}} \hat{K}_{n}^{\prime}$, and given $\lambda, \lambda^{\prime} \in \mathrm{C}$, then $\lambda \hat{K}_{\infty}+\lambda^{\prime} \hat{K}_{\infty}^{\prime}=\lim _{n \in \mathcal{N}}\left(\lambda \hat{K}_{n}+\lambda^{\prime} \hat{K}_{n}^{\prime}\right)$ and $\hat{K}_{\infty} \hat{K}_{\infty}^{\prime}=\lim _{n \in \mathcal{N}} \hat{K}_{n} \hat{K}_{n}^{\prime}$.

Proof: This is obvious.

Theorem 2.4: Given any bounded $\hat{K}_{\infty}$ on $\mathcal{L}_{\infty}$, then there exist bounded operators $\hat{K}_{n}$ on each $\mathcal{L}_{n}$ such that $\hat{K}_{\infty}=\lim _{n \in \mathcal{N}} \hat{K}_{n}$ and $\left\|\hat{K}_{n}\right\|=\left\|\hat{K}_{\infty}\right\|$ for all $n \in \mathcal{N}$.

Proof: Let $\mathcal{B}_{\infty}$ and $\mathcal{B}_{n}$ be as above. We define

$$
K_{j, k}=\left\langle\beta_{j, \infty} \mid \hat{K}_{\infty} \beta_{k, \infty}\right\rangle
$$

for all $j, k \in \mathbb{N}$. (Note that $K_{j, k}=0$ unless $j$ and $k$ both belong to $J_{\infty}$.) On each space $\mathcal{L}_{n}$, we define an operator $\hat{K}_{n}^{\prime}$ annihilating $\mathcal{L}_{n}^{\perp}$ and such that

$$
K_{j, k}=\left\langle\beta_{j, n} \mid \hat{K}_{n}^{\prime} \beta_{k, n}\right\rangle
$$

for all $j, k \in J_{n}$. Consider vectors $\psi_{\infty} \in \mathcal{L}_{\infty}$ and $\psi_{n} \in \mathcal{L}_{n}$ such that $\psi_{\infty}=\lim _{n} \psi_{n}$. Let the coefficients $c_{j, \infty}$ and $c_{j, n}$ be as above. Then

$$
\left\|\hat{K}_{n}^{\prime} \psi_{n}\right\|^{2}=\sum_{j=0}^{\infty}\left|\sum_{k=0}^{\infty} K_{j, k} c_{k, n}\right|^{2} \leqslant\left\|\hat{K}_{\infty}\right\|^{2}\left\|\psi_{n}\right\|^{2} .
$$

So the norms $\left\|\hat{K}_{n}^{\prime}\right\|$ are bounded by $\left\|\hat{K}_{\infty}\right\|$. Given $\epsilon>0$, then there exists a positive integer $N$ and complex numbers $c_{0}, \ldots, c_{N-1}$ such that

$$
\sum_{j=0}^{N-1}\left|\sum_{k=0}^{N-1} K_{j, k} c_{k}\right|^{2} \geqslant\left(\left\|\hat{K}_{\infty}\right\|-\epsilon\right)^{2} \sum_{j=0}^{N-1}\left|c_{j}\right|^{2} .
$$

For sufficiently large $n \in \mathcal{N}$, we have $\{0, \ldots, N-1\} \cap J_{\infty} \subseteq J_{n}$, whereupon $\left\|\hat{K}_{n}^{\prime}\right\| \geqslant\left\|\hat{K}_{\infty}\right\|-\epsilon$. Therefore, $\left\|\hat{K}_{\infty}\right\|=\lim _{n \in \mathcal{N}}\left\|\hat{K}_{n}^{\prime}\right\|$.

We claim that $\hat{K}_{\infty}=\lim _{n} \hat{K}_{n}^{\prime}$. Let $\phi \in \mathcal{S}$. For each $n$, let $\phi_{n}:=\operatorname{res}_{n}(\phi)$. To prove the claim, it suffices to show that

$$
\left\langle\phi \mid \hat{K}_{\infty} \psi_{\infty}\right\rangle=\lim _{n}\left\langle\phi_{n} \mid \hat{K}_{n}^{\prime} \psi_{n}\right\rangle
$$

For each $j \in \mathbb{N}$, let $a_{j, \infty}:=\left\langle\beta_{j, \infty} \mid \phi\right\rangle$ and $a_{j, n}:=\left\langle\beta_{j, n} \mid \phi_{n}\right\rangle$. Thus

$$
\phi=\sum_{j=0}^{\infty} a_{j, \infty} \beta_{j, \infty} \quad \text { and } \quad \phi_{n}=\phi_{n}^{\perp}+\sum_{j=0}^{\infty} a_{j, n} \beta_{j, n},
$$

where $\psi_{n}^{\perp} \in \mathcal{L}_{n}^{\perp}$. We have

$$
\left\langle\phi_{n} \mid \hat{K}_{n}^{\prime} \psi_{n}\right\rangle=\sum_{j, k=0}^{\infty} \bar{a}_{j, n} K_{j, k} c_{k, n}
$$

and a similar equation holds for $\left\langle\phi \mid \hat{K}_{\infty} \psi_{n}\right\rangle$. (By absolute convergence properties, all the sums we consider can be rearranged.) We have

$$
\left|\left\langle\phi \mid \hat{K}_{\infty} \psi_{\infty}\right\rangle-\left\langle\phi_{n} \mid \hat{K}_{n}^{\prime} \psi_{n}\right\rangle\right| \leqslant\left|\sum_{j, k=0}^{\infty} \bar{a}_{j, \infty} K_{j, k}\left(c_{k, \infty}-c_{k, n}\right)\right|+\left|\sum_{j, k=0}^{\infty}\left(\bar{a}_{j, \infty}-\bar{a}_{j, n}\right) K_{j, k} c_{k, n}\right| .
$$

(Using the boundedness of $\hat{K}_{\infty}$, it is easy to check that these sums are absolutely convergent.) Letting $C$ be an upper bound for the norms $\left\|\psi_{n}\right\|$, then 


$$
\sum_{j=0}^{\infty}\left|\sum_{k=0}^{\infty} K_{j, k} c_{k, n}\right|^{2} \leqslant C^{2}\left\|\hat{K}_{\infty}\right\|^{2}
$$

for sufficiently large $n$. Part of Ref. 6, Lemma 3.3, says that $\Sigma_{j=0}^{\infty}\left|a_{j, \infty}-a_{j, n}\right|^{2} \leqslant \epsilon^{2}$ for sufficiently large $n$. Hence

$$
\left|\sum_{j, k=0}^{\infty}\left(\bar{a}_{j, \infty}-\bar{a}_{j, n}\right) \hat{K}_{j, k} c_{k, n}\right| \leqslant \epsilon C\left\|\hat{K}_{\infty}\right\| .
$$

We may insist that $C \geqslant\left\|\psi_{\infty}\right\|$. Thereupon,

$$
\sum_{j=0}^{\infty}\left|\sum_{k=0}^{\infty} K_{j, k}\left(c_{k, \infty}-c_{k, n}\right)\right|^{2} \leqslant 4 C^{2}\left\|\hat{K}_{\infty}\right\|^{2}
$$

for sufficiently large $n$. The series $\sum_{j=0}^{\infty}\left|a_{j, \infty}\right|^{2}$ converges (to $\|\phi\|^{2}$ ), so there exists a positive integer $M$ such that $\sum_{j=M}^{\infty}\left|a_{j, \infty}\right|^{2} \leqslant \epsilon^{2}$. We have

$$
\left|\sum_{j=M}^{\infty} \sum_{k=0}^{\infty} \bar{a}_{j, \infty} K_{j, k}\left(c_{k, \infty}-c_{k, n}\right)\right| \leqslant 2 \epsilon C\left\|\hat{K}_{\infty}\right\|
$$

for large $n$. To prove the claim, it now suffices to show that

$$
\left|\sum_{j=0}^{M-1} \sum_{k=0}^{\infty} \bar{a}_{j, \infty} K_{j, k}\left(c_{k, \infty}-c_{k, n}\right)\right|=O(\epsilon) .
$$

Let $j \in \mathbb{N}$. Suppose there exists some $\delta>0$ such that, for every positive integer $L$, there exist complex numbers $c_{L}, c_{L+1}, \ldots$ satisfying

$$
\sum_{k=L}^{\infty}\left|c_{k}\right|^{2} \leqslant 1 \quad \text { and } \quad\left|\sum_{k=L}^{\infty} K_{j, k} c_{k}\right|>\delta .
$$

Then there exist complex numbers $c_{0}, c_{1}, \ldots$ and integers $0=L_{0}<L_{1}<\cdots$ such that each $K_{j, k} c_{k}$ is a non-negative real, and

$$
\sum_{k=L_{r-1}}^{L_{r}-1}\left|c_{k}\right|^{2} \leqslant \frac{1}{n^{2}} \text { and } \sum_{k=L_{r-1}}^{L_{r}-1} K_{j, k} c_{k}>\frac{\delta}{2 n}
$$

for all positive integers $r$. The series $\sum_{k=0}^{\infty}\left|c_{k}\right|^{2}$ converges while the series $\sum_{k=0}^{\infty} K_{j, k} c_{k}$ diverges. This contradicts the boundedness of $\hat{K}_{\infty}$. We deduce that, for any positive real $B$, there exists a positive integer $L$ such that, for all complex numbers $c_{L}, c_{L+1}, \ldots$ satisfying $\Sigma_{k=L}^{\infty}\left|c_{k}\right|^{2} \leqslant B$, we have $\left|\sum_{k=L}^{\infty} K_{j, k} c_{k}\right| \leqslant \epsilon / M$. For large $n$, we have $\sum_{k=0}^{\infty}\left|c_{k, \infty}-c_{k, n}\right|^{2} \leqslant 4 C^{2}$. So there exists a positive integer $L$ such that, for large $n$, and for all $j<M$, we have

$$
\left|\sum_{k=L}^{\infty} K_{j, k}\left(c_{k, \infty}-c_{k, n}\right)\right| \leqslant \epsilon / M .
$$

Each $\left|a_{j, \infty}\right| \leqslant\|\phi\|$, so

$$
\left|\sum_{j=0}^{M-1} \sum_{k=L}^{\infty} \bar{a}_{j, \infty} K_{j, k}\left(c_{k, \infty}-c_{k, n}\right)\right| \leqslant \epsilon\|\phi\|
$$

for large $n$. The claim will follow when we have shown that 


$$
\left|\sum_{j=0}^{M-1} \sum_{k=0}^{L-1} \bar{a}_{j, \infty} K_{j, k}\left(c_{k, \infty}-c_{k, n}\right)\right|=O(\epsilon)
$$

for large $n$. By Theorem 2.2, $c_{k, \infty}=\lim _{n \in \mathcal{N}} c_{k, n}$. The claim is established.

To finish the argument, we must replace the operators $\hat{K}_{n}^{\prime}$ with operators $\hat{K}_{n}$ on $\mathcal{L}_{n}$ such that $\left\|\hat{K}_{n}\right\|=\left\|\hat{K}_{\infty}\right\|$ for all $n \in \mathcal{N}$. We may assume that $\left\|\hat{K}_{\infty}\right\|=1$. From the first paragraph of the argument, $\left\|\hat{K}_{n}^{\prime}\right\|$ converges to 1 . So $\hat{K}_{n} \neq 0$ for large $n$. When $\hat{K}_{n}^{\prime} \neq 0$, we put $\hat{K}_{n}=\hat{K}_{n}^{\prime} /\left\|\hat{K}_{n}^{\prime}\right\|$, otherwise we put $\hat{K}_{n}=\hat{1}$. Then each $\left\|\hat{K}_{n}\right\|=1$, and $\left\|\hat{K}_{n}-\hat{K}_{n}^{\prime}\right\| \rightarrow 0$. Since the norms $\left\|\psi_{n}\right\|$ are bounded, $\left\|\hat{K}_{n} \psi_{n}-\hat{K}_{n}^{\prime} \psi_{n}\right\| \rightarrow 0$. It was shown in Ref. 6, Remark 2.3, that, for $\theta_{\infty} \in \mathcal{L}_{\infty}$ and $\theta_{n}, \chi_{n} \in \mathcal{L}_{n}$ satisfying $\theta_{\infty}=\lim _{n \in \mathcal{N}} \theta_{n}$ and $\lim _{n \in \mathcal{N}}\left\|\theta_{n}-\chi_{n}\right\|=0$, we have $\theta_{\infty}=\lim _{n \in \mathcal{N}} \chi_{n}$. Therefore, $\hat{K}_{\infty} \psi_{\infty}$ $=\lim _{n \in \mathcal{N}} \hat{K}_{n} \psi_{n}$.

Corollary 2.5: Given any bounded Hermitian operator $\hat{H}_{\infty}$ on $\mathcal{L}_{\infty}$, then there exist bounded Hermitian operators $\hat{H}_{n}$ on each $\mathcal{L}_{n}$ such that $\hat{H}_{\infty}=\lim _{n \in \mathcal{N}} \hat{H}_{n}$ and $\left\|\hat{H}_{n}\right\|=\left\|\hat{H}_{\infty}\right\|$ for each $n$ $\in \mathcal{N}$.

Proof: In the proof of Theorem 2.4, if $\hat{K}_{\infty}$ is Hermitian, then so is each $\hat{K}_{n}$.

In order to accommodate the possibility of working with a compound of several quantum stochastic processes (for example, a quantum system with several particles), we must discuss tensor products of inductive resolutions, and we must show how the limits of vectors and operators are compatible with the tensor product. Let $\mathcal{L}_{\infty}^{\prime}$ be a Hilbert space, and let $\mathcal{S}^{\prime}$ be a dense subspace of $\mathcal{L}_{\infty}^{\prime}$. For each $n \in \mathcal{N}$, let $\mathcal{L}_{n}^{\prime}$ be a Hilbert space, and let res ${ }_{n}^{\prime}: \mathcal{S}^{\prime} \rightarrow \mathcal{L}_{n}$ be restriction maps. Then $\mathcal{L}_{\infty} \otimes \mathcal{L}_{\infty}^{\prime}$ has an inductive resolution with restriction maps $\operatorname{res}_{n} \otimes \operatorname{res}_{n}^{\prime}: \mathcal{S} \otimes \mathcal{S}^{\prime} \rightarrow \mathcal{L}_{n} \otimes \mathcal{L}_{n}^{\prime}$. Given limits of vectors $\psi_{\infty}=\lim _{n} \psi_{n}$ and $\psi_{\infty}^{\prime}=\lim _{n} \psi_{n}^{\prime}$ in $\mathcal{L}_{\infty}$ and $\mathcal{L}_{\infty}^{\prime}$, respectively, it is clear that we have a limit of vectors $\psi_{\infty} \otimes \psi_{\infty}^{\prime}=\lim _{n} \psi_{n} \otimes \psi_{n}^{\prime}$. By considering orthonormal coordinates and applying Ref. 6, Theorem 3.4, it is easy to check that limits of bounded operators preserve tensor products in the same way. (Warning: we are not invoking Ref. 6, Theorem 3.4, gratuitously. Not every sequence in $\mathcal{L}_{n} \otimes \mathcal{L}_{n}^{\prime}$ converging to $\psi_{\infty} \otimes \psi_{\infty}^{\prime}$ has terms of the form $\psi_{n} \otimes \psi_{n}^{\prime}$.) These (rather trivial) remarks show that the limits behave well in the (rather banal) case of a fixed finite number of noninteracting processes. Presumably, they also behave well with respect to symmetric and antisymmetric tensor products, and with respect to the construction of free, symmetric, and antisymmetric Fock spaces (see Ref. 9, Chap. II). We leave that matter for further research.

\section{CONVERGENCE OF QUANTUM SYSTEMS}

Recall that a family $\{\hat{K}(t): t \in \mathbb{R}\}$ of operators on a Hilbert space $\mathcal{L}$ is said to be strongly continuous provided each $\hat{K}(t)$ has domain $\mathcal{L}$ and, for all $\psi \in \mathcal{L}$, the function $\mathbb{R} \rightarrow \mathcal{L}$ given by $t \mapsto \hat{K}(t) \psi$ is continuous. If, furthermore, $\hat{K}(0)=\hat{1}$ and each $\hat{K}(t)$ is bounded, then we call $\{\hat{K}(t): t \in \mathbb{R}\}$ a quantum system on $\mathcal{L}$. In that case, we sometimes consider a family of vectors $\{\psi(t): t \in \mathbb{R}\}$ such that

$$
\psi(t)=\hat{K}(t) \psi(0) .
$$

A quantum system $\mathcal{U}=\{\hat{U}(t): t \in \mathbb{R}\}$ on $\mathcal{L}$ is said to be unitary provided each operator $\hat{U}(t)$ is unitary. If, furthermore,

$$
\hat{U}(t) \hat{U}\left(t^{\prime}\right)=\hat{U}\left(t+t^{\prime}\right)
$$

for all $t, t^{\prime} \in \mathbb{R}$, then we say that $\mathcal{U}$ is conservative.

The boundedness condition in our general definition of a quantum system is somewhat artificial, but convenient for our purposes. Our main concern is with conservative systems, and these have been thoroughly studied in various contexts and from various perspectives. For a detailed 
introduction to conservative systems as quantum stochastic systems, see Ref. 9, Chap. 1. Let us recall some well-known properties of conservative systems (introducing some notation that will be convenient in the proof of Theorem 3.5).

Suppose that $\mathcal{U}$ is conservative. Stone's theorem asserts that there exists a unique Hermitian operator $\hat{H}$ on $\mathcal{L}$ such that

$$
U(t)=\exp (-i \hat{H} t)
$$

We call $\hat{H}$ the Hamiltonian for $\mathcal{U}$. Conversely, every Hermitian operator on $\mathcal{L}$ is the Hamiltonian of a conservative quantum system. The bijective correspondence $\hat{H} \leftrightarrow \mathcal{U}$ allows us to characterize conservative quantum systems by the Schrödinger equation

$$
i \frac{d}{d t} \psi(t)=\hat{H} \psi(t)
$$

For the sake of rigor, we must mention that, as a definition,

$$
\exp (-i \hat{H} t):=\int_{-\infty}^{\infty} e^{-i t s} d E(s)
$$

where $E$ is the spectral family for $\hat{H}$. The notation on the right-hand side is as in Ref. 10, Chap. 7. It may be worth explaining what this equation tells us. Introducing some notation that will be of use in the proof of Theorem 3.5, let us consider an integer $m$, and write $\hat{E}_{m}$ for the orthogonal projection on $\mathcal{L}$ associated with $E$ and the half-open interval [m,m+1). [Intuitively, we might think of $\hat{E}_{m}$ as the projection to the subspace $\hat{E}_{m} \mathcal{L}$ of $\mathcal{L}$ spanned by those "eigenvectors" whose "eigenvalues" are at least $m$ and less than $m+1$. The operator $\hat{H}$ restricts to an operator on each subspace $\hat{E}_{m} \mathcal{L}$. Vaguely, we might think of $\hat{E}_{m}$ as a kind of "eigenspace," whose associated "eigenvalue" is spread across the interval $[m, m+1)$.] Any vector in $\mathcal{L}$ is a sum of vectors belonging to the spaces $\hat{E}_{m} \mathcal{L}$, so the unitary operator $\exp (-i \hat{H} t)$ is determined by the condition that it restricts to an operator on $\hat{E}_{m} \mathcal{L}$ given by

$$
\exp (-i \hat{H} t) \psi=\sum_{l=0}^{\infty} \frac{(-i \hat{H} t)^{l}}{l !} \psi
$$

for all $\psi \in \hat{E}_{m} \mathcal{L}$. (The series converges because $\hat{H}$ restricts to a bounded operator on $\hat{E}_{m} \mathcal{L}$.)

Stone's theorem may be found in Ref. 10, Theorem 7.38. The bijectivity of the correspondence $\hat{H} \leftrightarrow \mathcal{U}$ is given in Ref. 15, Theorem 7.37. See also Ref. 9, Theorem 13.1.

Given a quantum system $\mathcal{K}_{\infty}=\left\{\hat{K}_{\infty}(t): t \in \mathbb{R}\right\}$ on $\mathcal{L}_{\infty}$, and quantum systems $\mathcal{K}_{n}=\left\{\hat{K}_{n}(t): t\right.$ $\in \mathbb{R}\}$ on $\mathcal{L}_{n}$ for sufficiently large $n \in \mathcal{N}$, we say that $\left(\mathcal{K}_{n}\right)_{n}$ converges to $\mathcal{K}_{\infty}$ provided

$$
\hat{K}_{\infty}(t)=\lim _{n \in \mathcal{N}} \hat{K}_{n}(t)
$$

for all $t \in \mathbb{R}$. Obviously, $\left(\mathcal{K}_{n}\right)_{n}$ converges to at most one quantum system on $\mathcal{L}_{\infty}$. When $\left(\mathcal{K}_{n}\right)_{n}$ converges to $\mathcal{K}_{\infty}$, we call $\mathcal{K}_{\infty}$ the limit of $\left(\mathcal{K}_{n}\right)_{n}$, and we write $\mathcal{K}_{\infty}=\lim _{n \in \mathcal{N}} \mathcal{K}_{n}$.

Remark 3.1: Let $\mathcal{K}_{\infty}=\left\{\hat{K}_{\infty}(t): t \in \mathbb{R}\right\}$ and $\mathcal{K}_{n}=\left\{\hat{K}_{n}(t): t \in \mathbb{R}\right\}$, respectively, be quantum systems on $\mathcal{L}_{\infty}$ and on each $\mathcal{L}_{n}$. Write $\psi_{\infty}(t)=\hat{K}_{\infty}(t) \psi_{\infty}(0)$ and $\psi_{n}(t)=\hat{K}_{n}(t) \psi_{n}(0)$. Then we have a limit of quantum systems $\mathcal{K}_{\infty}=\lim _{n \in \mathcal{N}} \mathcal{K}_{n}$ if and only if, given any initial state vectors $\psi_{\infty}(0)$ in $\mathcal{L}_{\infty}$ and $\psi_{n}(0)$ in each $\mathcal{L}_{n}$ with $\psi_{\infty}(0)=\lim _{n \in \mathcal{N}}\left(\psi_{n}(0)\right)$, and writing $\psi_{\infty}(t)=\hat{K}_{\infty}(t) \psi_{\infty}(0)$ and $\psi_{n}(t)$ $=\hat{K}_{n}(t) \psi_{n}(0)$, we have $\psi_{\infty}(t)=\lim _{n \in \mathcal{N}} \psi_{n}(t)$ for all $t \in \mathbb{R}$.

Proof: This is obvious. 
In particular, Remark 3.1 tells us that if the limit holds for the quantum systems and for the initial vectors, then the limit holds for all the time-evolved vectors. In case this seems counterintuitive, we point out that, if $\psi_{n}(t)$ is to be a "good approximation" to $\psi_{\infty}(t)$, one should first fix $t$, and then choose $n$.

Theorem 3.2: Any quantum system on $\mathcal{L}_{\infty}$ is the limit of a sequence of quantum systems on the spaces $\mathcal{L}_{n}$. define

Proof: Let $\mathcal{K}_{\infty}=\left\{\hat{K}_{\infty}(t): t \in \mathbb{R}\right\}$ be a quantum system on $\mathcal{L}_{\infty}$. For each $t \in \mathbb{R}$, and $j, k \in \mathbb{N}$, we

$$
K_{j, k}(t):=\left\langle\beta_{j, \infty} \mid \hat{K}_{\infty}(t) \beta_{k, \infty}\right\rangle
$$

Let $\hat{K}_{n}(t)$ be the operator in $\mathcal{L}_{n}$ constructed from the matrix entries $K_{j, k}(t)$ as in the proof of Theorem 2.4. Let $\mathcal{K}_{n}=\left\{\hat{K}_{n}(t): t \in \mathbb{R}\right\}$. Using the condition that $\mathcal{K}_{\infty}$ is strongly continuous, it is easy to check that each $\mathcal{K}_{n}$ is strongly continuous.

Proposition 3.3: Let $\hat{H}_{\infty}$ and each $\hat{H}_{n}$ be bounded Hermitian operators on $\mathcal{L}_{\infty}$ and $\mathcal{L}_{n}$, respectively, and suppose that the norms $\left\|\hat{H}_{n}\right\|$ are bounded. Let $\mathcal{U}_{\infty}$ and each $\mathcal{U}_{n}$ be the conservative systems with Hamiltonians $\hat{H}_{\infty}$ and $\hat{H}_{n}$, respectively. Then $\mathcal{U}_{\infty}=\lim _{n \in \mathcal{N}} \mathcal{U}_{n}$ if and only if $\hat{H}_{\infty}=\lim _{n \in \mathcal{N}} \hat{H}_{n}$.

Proof: Write $\mathcal{U}_{\infty}=\left\{\hat{U}_{\infty}(t): t \in \mathbb{R}\right\}$ and $\mathcal{U}_{n}=\left\{\hat{U}_{n}(t): t \in \mathbb{R}\right\}$. For $m \in \mathbb{N}$, let

$$
\hat{K}_{m, \infty}(t):=\sum_{k=0}^{m} \frac{\left(-i \hat{H}_{\infty} t\right)^{k}}{k !} \text { and } \quad \hat{K}_{m, n}(t):=\sum_{k=0}^{m} \frac{\left(-i \hat{H}_{n} t\right)^{k}}{k !} .
$$

Then $\hat{U}_{\infty}(t)=\lim _{m \rightarrow \infty} \hat{K}_{m, \infty}(t)$ and $\hat{U}_{n}(t)=\lim _{m \rightarrow \infty} \hat{K}_{m, n}(t)$.

Let $\epsilon>0$. Consider vectors $\phi \in \mathcal{S}$ and $\psi_{\infty} \in \mathcal{L}_{\infty}$ and $\psi_{n} \in \mathcal{L}_{n}$ such that $\psi_{\infty}=\lim _{n} \psi_{n}$. Write $\phi_{n}=\operatorname{res}_{n}(\phi)$. Let $A$ be an upper bound for $\|\phi\|$ and $\left\|\phi_{n}\right\|$. Let $B$ be an upper bound for $\left\|\hat{H}_{\infty}\right\|$ and $\left\|\hat{H}_{n}\right\|$. Let $C$ be an upper bound for $\left\|\psi_{\infty}\right\|$ and $\left\|\psi_{n}\right\|$. Choose $m$ such that

$$
2 A C \sum_{k=m}^{\infty}|B t|^{k} / k ! \leqslant \epsilon
$$

Then $\left\|\hat{U}_{\infty}(t)-K_{m, \infty}(t)\right\| \leqslant \epsilon / 2 A C \geqslant\left\|\hat{U}_{n}(t)-K_{m, n}(t)\right\|$ for sufficiently large $n$. Hence

$$
\left|\left\langle\phi\left|\hat{U}_{\infty}(t)-\hat{K}_{m, \infty}(t)\right| \psi_{\infty}\right\rangle-\left\langle\phi_{n}\left|\hat{U}_{n}-\hat{K}_{m, n}(t)\right| \psi_{n}\right\rangle\right| \leqslant \epsilon .
$$

If $\hat{H}_{\infty}=\lim _{n} \hat{H}_{n}$, then, by Remark 2.3, $\hat{K}_{m, \infty}=\lim _{n} \hat{K}_{m, n}$, hence $\hat{U}_{\infty}(t) \psi_{\infty}=\lim _{n \in \mathcal{N}} \hat{U}_{n}(t) \psi_{n}$.

Conversely, suppose that $\hat{U}_{\infty}(t) \psi_{\infty}=\lim _{n \in \mathcal{N}} \hat{U}_{n}(t) \psi_{n}$. Given $t$, we can put $\epsilon=t^{2} / 2$ (and then choose $m$ ), where

$$
\left|\left\langle\phi \mid \hat{K}_{m, \infty}(t) \psi_{\infty}\right\rangle-\left\langle\phi_{n} \mid \hat{K}_{m, n}(t) \psi_{n}\right\rangle\right|=O\left(t^{2}\right)
$$

for sufficiently large $n$. Equating coefficients of $t$ (the sums $\sum_{k=0}^{m}\left|\hat{H}_{n} t\right|^{k} / k$ ! and the similar sum for $\hat{H}_{\infty}$ are bounded by $\left.e^{B|t|}\right)$, we obtain $\hat{H}_{\infty} \psi_{\infty}=\lim _{n} \hat{H}_{n} \psi_{n}$.

Corollary 3.4: Let $\mathcal{U}_{\infty}$ be a conservative system on $\mathcal{L}_{\infty}$ with bounded Hamiltonian $\hat{H}_{\infty}$. Then there exist conservative systems $\mathcal{U}_{n}$ on $\mathcal{L}_{n}$ with bounded Hamiltonians $\hat{H}_{n}$ such that $\mathcal{U}_{\infty}$ $=\lim _{n \in \mathcal{N}} \mathcal{U}_{n}$ and $\hat{H}_{\infty}=\lim _{n \in \mathcal{N}} \hat{H}_{n}$.

Proof: This is immediate from Corollary 2.5 and Proposition 3.3.

Theorem 3.5: Any conservative system on $\mathcal{L}_{\infty}$ is the limit of a sequence of conservative systems on the spaces $\mathcal{L}_{n}$. 
Proof: Let $\mathcal{U}_{\infty}=\left\{\hat{U}_{\infty}(t): t \in \mathbb{R}\right\}$ be a conservative system on $\mathcal{L}_{\infty}$, let $\hat{H}_{\infty}$ be the Hamiltonian for $\mathcal{U}_{\infty}$, and let $E$ be the spectral family for $\hat{H}_{\infty}$. For each $m \in \mathbb{Z}$, let $\hat{E}_{m}$ be the orthogonal projection as above, and let $\mathcal{L}_{m, \infty}=\hat{E}_{m} \mathcal{L}_{\infty}$. The Hermitian operator $\hat{H}_{\infty}$ restricts to a Hermitian operator $\hat{H}_{m, \infty}$ on $\mathcal{L}_{m, \infty}$. Let $\mathcal{U}_{m, \infty}=\left\{\hat{U}_{m, \infty}(t): t \in \mathbb{R}\right\}$ be the conservative system on $\mathcal{L}_{m, \infty}$ with Hamiltonian $\hat{H}_{m, \infty}$. Any vector $\psi_{\infty} \in \mathcal{L}_{\infty}$ has a unique decomposition as a sum

$$
\psi_{\infty}=\sum_{m \in \mathbb{Z}} \psi_{m, \infty}
$$

where each $\psi_{m, \infty} \in \mathcal{L}_{m, \infty}$. We have $\hat{H}_{\infty} \psi_{m, \infty}=\hat{H}_{m, \infty} \psi_{m, \infty}$ and

$$
\hat{U}_{\infty}(t) \psi_{\infty}=\sum_{m \in Z} \hat{U}_{m, \infty}(t) \psi_{m, \infty}
$$

It is easy to see that there exists an enumerated orthonormal basis $\mathcal{B}_{\infty}=\left\{\beta_{j, \infty}: j \in J_{\infty}\right\}$ such that each $\beta_{j, \infty}$ belongs to one of the subspaces $\mathcal{L}_{m, \infty}$. The enumerated orthonormal sets $\mathcal{B}_{n}$, as in Sec. II, may be chosen such that each $J_{n} \subseteq J_{\infty}$. For each $m \in \mathbb{Z}$, let

$$
J_{\infty}(m):=\left\{j \in J_{\infty}: \beta_{j, \infty} \in \mathcal{L}_{m, \infty}\right\} \quad \text { and } \quad J_{n}(m):=J_{n} \cap J_{\infty}(m) .
$$

Let $\mathcal{L}_{m, n}$ be the subspace of $\mathcal{L}_{n}$ spanned by the vectors $\beta_{j, n}$ such that $j \in J_{n}(m)$. Any vector $\chi_{n}$ $\in \mathcal{L}_{n}$ has a unique decomposition as a sum

$$
\chi_{n}=\chi_{n}^{\perp}+\sum_{m \in Z} \chi_{m, n}
$$

where $\chi_{n}^{\perp} \in \mathcal{L}_{n}^{\perp}$, and each $\chi_{m, n} \in \mathcal{L}_{m, n}$. For $j, k \in J_{\infty}$, let

$$
H_{j, k}=\left\langle\beta_{j, \infty} \mid \hat{H}_{\infty} \beta_{k, \infty}\right\rangle .
$$

Note that $H_{j, k}=\overline{H_{k, j}}$, and $H_{j, k}=0$ unless $j, k \in J_{\infty}(m)$ for some $m \in \mathbb{Z}$. Let $\hat{H}_{m, n}$ be the Hermitian operator on $\mathcal{L}_{m, n}$ such that

$$
H_{j, k}=\left\langle\beta_{j, n} \mid \hat{H}_{m, n} \beta_{k, n}\right\rangle
$$

for $j, k \in J_{n}(m)$. Let $\mathcal{U}_{m, n}=\left\{\hat{U}_{m, n}(t): t \in \mathbb{R}\right\}$ be the conservative system on $\mathcal{L}_{m, n}$ with Hamiltonian $\hat{H}_{m, n}$. Let $\hat{H}_{n}$ be the Hermitian operator on $\mathcal{L}_{n}$ such that $\hat{H}_{n} \chi_{n}^{\perp}=0$ and $\hat{H}_{n} \chi_{m, n}=\hat{H}_{m, n} \chi_{m, n}$. Let $\mathcal{U}_{n}=\left\{\hat{U}_{n}(t): t \in \mathbb{R}\right\}$ be the conservative system on $\mathcal{L}_{n}$ with Hamiltonian $\hat{H}_{n}$. Then

$$
\hat{U}_{n}(t) \chi_{n}=\chi_{n}^{\perp}+\sum_{m \in Z} \hat{U}_{m, n}(t) \chi_{m, n}
$$

We are to show that $\hat{U}_{\infty}(t)=\lim _{n \in \mathcal{N}} \hat{U}_{n}(t)$ for all $t \in \mathbb{R}$.

For each $n \in \mathcal{N}$, let $\psi_{n} \in \mathcal{L}_{n}$, and suppose that $\psi_{\infty}=\lim _{n \in \mathcal{N}} \psi_{n}$. Write

$$
\psi_{\infty}=\sum_{j=0}^{\infty} c_{j, \infty} \beta_{j, \infty} \quad \text { and } \quad \psi_{n}=\psi_{n}^{\perp}+\sum_{j=0}^{\infty} c_{j, n} \beta_{j, n}
$$

as in Sec. II. Fix $t \in \mathbb{R}$, and let $\theta_{\infty}=\hat{U}_{\infty}(t) \psi_{\infty}$ and $\theta_{n}=\hat{U}_{n} \psi_{n}$. We are to show that $\theta_{\infty}$ $=\lim _{n} \theta_{n}$. Write 


$$
\theta_{\infty}=\sum_{j=0}^{\infty} d_{j, \infty} \beta_{j, \infty} \quad \text { and } \quad \theta_{n}=\theta_{n}^{\perp}+\sum_{j=0}^{\infty} d_{j, n} \beta_{j, n}
$$

as we did for $\psi_{\infty}$ and $\psi_{n}$. The norms $\left\|\theta_{n}\right\|=\left\|\psi_{n}\right\|$ are bounded. So, by Theorem 2.2, we are to show that $d_{j, \infty}=\lim _{n} d_{j, n}$ for all $j \in J_{\infty}$. Fix $j \in J_{\infty}$, and let $m$ be such that $j \in J_{\infty}(m)$. We have

$$
d_{j, \infty}=\sum_{k \in J_{\infty}(m)}\left\langle\beta_{j, \infty} \mid \hat{U}_{m, \infty}(t) \beta_{k, \infty}\right\rangle c_{k, \infty} .
$$

The equation still holds with the symbol $n$ instead of the symbol $\infty$. Replacing $\hat{H}_{\infty}$ with the Hermitian operator $\hat{E}_{m} \hat{H}_{\infty}=\hat{H}_{\infty} \hat{E}_{m}$ does not change $\hat{H}_{m, \infty}$ or $\hat{H}_{m, n}$, so it does not change $\hat{U}_{m, \infty}$ or $\hat{U}_{n, m}$. So it does not change $d_{j, \infty}$ or $d_{j, n}$. Therefore, we may assume that $\hat{H}_{m^{\prime}, \infty}=0$ for all integers $m^{\prime} \neq m$. Hence $\hat{H}_{m^{\prime}, n}=0$ for all $m^{\prime} \neq m$ and all $n \in \mathcal{N}$. But now $\hat{H}_{\infty}$ is bounded, indeed $\left\|\hat{H}_{\infty}\right\|$ $\leqslant|m|+1$. Furthermore, the operators $\hat{H}_{n}$ are constructed from $\hat{H}_{\infty}$ just as the operators $\hat{K}_{n}^{\prime}$ were constructed from $\hat{K}_{\infty}$ in the proof of Theorem 2.4. So $\hat{H}_{\infty}=\lim _{n \in \mathcal{N}} \hat{H}_{n}$. Thanks to Proposition 3.3, the argument is now complete.

Corollary 3.6: Any unitary operator on $\mathcal{L}_{\infty}$ is the limit of a sequence of unitary operators on the spaces $\mathcal{L}_{n}$.

Proof: Given a unitary operator $\hat{U}_{\infty}$ on $\mathcal{L}_{\infty}$, then by Ref. 10 Exercise 7.50, there exists a conservative system $\left\{\hat{U}_{\infty}(t): t \in \mathbb{R}\right\}$ such that $\hat{U}_{\infty}=\hat{U}_{\infty}(1)$. Theorem 3.5 now gives the assertion. $\square$

A more direct way to demonstrate Corollary 3.6 is to adapt the proof of Theorem 2.4, using the Gram-Schmidt process to modify the columns of the matrices $\left(K_{j, k}\right)_{j, k \in J_{n}}$. The argument is fairly routine, although it is complicated by the need to make some arbitrary choices when the Gram-Schmidt process terminates prematurely.

The existence results above can be interpreted as saying that, in principle, any "continuum" system (of a particular kind) is the limit of a sequence of "discrete" systems (of the same kind). The next result provides one way of actually recognizing that a given "continuum" system is the limit of a given sequence of "discrete" systems.

Proposition 3.7: Let $\mathcal{U}_{\infty}=\left\{\hat{U}_{\infty}(t): t \in \mathbb{R}\right\}$ be a conservative system on $\mathcal{L}_{\infty}$, and for each $n$ $\in \mathcal{N}$, let $\mathcal{U}_{n}=\left\{\hat{U}_{n}(t): t \in \mathbb{R}\right\}$ be a conservative system on $\mathcal{L}_{n}$. Let $\hat{H}_{\infty}$ and $\hat{H}_{n}$, respectively, be the Hamiltonians. Let $\mathcal{B}_{\infty}$ and $\mathcal{B}_{n}$ be as in Sec. II. Suppose that, for each $j \in J_{\infty}$, there exists a real $\lambda_{j, \infty}$ such that

$$
\hat{H}_{\infty} \beta_{j, \infty}=\lambda_{j, \infty} \beta_{j, \infty} .
$$

Suppose also that, for sufficiently large $n$, there exist reals $\lambda_{j, n}$ such that

$$
\hat{H}_{n} \beta_{j, n}=\lambda_{j, n} \beta_{j, n} .
$$

Then $\mathcal{U}_{\infty}=\lim _{n \in \mathcal{N}} \mathcal{U}_{n}$ if and only if $\lambda_{j, \infty}=\lim _{n \in \mathcal{N}} \lambda_{j, n}$ for all $j \in J_{\infty}$.

Proof: This follows quickly from Theorem 2.2.

Proposition 3.7 yields an alternative (and very easy) proof of Theorem 3.5 in the special case of a conservative system on $\mathcal{L}_{\infty}$ with a diagonalizable Hamiltonian.

\section{SOME EXAMPLES OF CONTINUUM LIMITS OF DISCRETE SYSTEMS}

In all the examples to follow, we shall apply Proposition 3.7 to show that the given "continuum" system is the limit of the given sequence of "discrete" systems. Each of the inductive resolutions is a sample-point inductive resolution, as in Ref. 6, Examples 2.A-2.F. Sample-point inductive resolutions are examined also in Ref. 7.

Example 4.A: The circular rotor. The rotor, in one dimension, is a model for a particle moving freely on a circle. Classically, the energy is proportional to the square of the angular momentum. 
Let $\mathcal{S}$ be the space of smooth functions $\phi: \mathrm{R} \rightarrow \mathrm{C}$ such that $\phi$ has period unity and $\phi$ is squareintegrable on a bounded domain. The inner product on $\mathcal{S}$ is given by integration over an interval of length unity. Making a suitable choice of units, the Hamiltonian $\hat{H}_{\infty}$ of the rotor has domain $\mathcal{S}$ and satisfies

$$
\hat{H}_{\infty} \phi(x)=-d^{2} \phi(x) / d x^{2}
$$

for $\phi \in \mathcal{S}$ and $x \in \mathbb{R}$. The completion $\mathcal{L}_{\infty}$ of $\mathcal{S}$ has an orthonormal basis $\mathcal{B}_{\infty}=\left\{\beta_{j, \infty}: j \in \mathbb{N}\right\}$ given by

$$
\beta_{j, \infty}(x)=\left\{\begin{array}{l}
\sqrt{2} \cos (\pi j x) \quad \text { if } j \text { is even, } \\
\sqrt{2} \sin (\pi(j+1) x) \text { if } j \text { is odd. }
\end{array}\right.
$$

It is easy to check that $\mathcal{B}_{\infty}$ diagonalizes $\mathcal{H}_{\infty}$, indeed, $\hat{H}_{\infty} \beta_{j, \infty}=\lambda_{j, \infty} \beta_{j, \infty}$, where

$$
\lambda_{j, \infty}=\left\{\begin{array}{l}
\pi^{2} j^{2} \text { if } j \text { is even, } \\
\pi^{2}(j+1)^{2} \text { if } j \text { is odd. }
\end{array}\right.
$$

Let $\mathcal{N}$ be the set of positive odd integers. For each $n \in \mathcal{N}$, let $\mathcal{L}_{n}$ be the $n$-dimensional inner product space consisting of the functions $\mathrm{Z} \rightarrow \mathrm{C}$ with period $n$. The inner product on $\mathcal{L}_{n}$ is given by summation over $n$ consecutive integers. We replace the differential operator $-d^{2} / d x^{2}$ with a difference operator $\hat{H}_{n}$ where

$$
\hat{H}_{n} \psi(X)=n^{2}(-\psi(X-1)+2 \psi(X)-\psi(X+1))
$$

for $\psi \in \mathcal{L}_{n}$ and $X \in \mathbb{Z}$. Given an integer $j$ with $0 \leqslant j \leqslant n-1$, we put

$$
\beta_{j, n}(X)= \begin{cases}\sqrt{2 / n} \cos (\pi j X / n) \quad \text { if } j & \text { is even, } \\ \sqrt{2 / n} \sin (\pi(j+1) X / n) & \text { if } j \text { is odd. }\end{cases}
$$

It is easy to check that $\left\{\beta_{j, n}: 0 \leqslant j \leqslant n-1\right\}$ is an orthonormal basis for $\mathcal{L}_{n}$ diagonalizing $\hat{H}_{n}$. Writing $\hat{H}_{n} \beta_{j, n}=\lambda_{j, n} \beta_{j, n}$, then

$$
\lambda_{j, n}=\left\{\begin{array}{l}
2 n^{2}(1-\cos (2 \pi j X / n)) \quad \text { if } j \text { is even, } \\
2 n^{2}(1-\cos (2 \pi(j+1) X / n)) \quad \text { if } j \text { is odd. }
\end{array}\right.
$$

Let $\mathcal{U}_{\infty}$ be the conservative system on $\mathcal{L}_{\infty}$ with Hamiltonian $\hat{H}_{\infty}$. For each $n \in \mathcal{N}$, let $\mathcal{U}_{n}$ be the conservative system on $\mathcal{L}_{n}$ with Hamiltonian $\hat{H}_{n}$. Of course, it is heuristically "obvious" that $\mathcal{U}_{\infty}$ is some kind of "limit" of $\mathcal{U}_{n}$, but in order to formulate this observation mathematically, we must realize $\left(\mathcal{L}_{n}\right)_{n}$ as an inductive resolution of $\mathcal{L}_{\infty}$. We define $\operatorname{res}_{n}: \mathcal{S} \rightarrow \mathcal{L}_{n}$ such that

$$
\operatorname{res}_{n}(\phi)(X)=\phi(X / n) / \sqrt{n}
$$

for $\phi \in \mathcal{S}$ and $X \in \mathbb{Z}$ with $-n / 2<X<n / 2$. It is easy to check that the sequence $\left(\mathcal{L}_{n}\right)_{n}$, equipped with the sequence $\left(\operatorname{res}_{n}\right)_{n}$, is indeed an inductive resolution of $\mathcal{L}_{\infty}$. (In fact, this is the precisely the one-dimensional case of Ref. 6, Example 2.F.) Given $j \in \mathbb{N}$, then, for all $n>j$, we have $\beta_{j, n}$ $=\operatorname{res}_{n}\left(\beta_{j, \infty}\right)$. Therefore, $\beta_{j, \infty}=\lim _{n \in \mathcal{N}} \beta_{j, n}$. Since $\lambda_{n, \infty}=\lim _{n \in \mathcal{N}} \lambda_{j, n}$, Proposition 3.7 tells us that

$$
\mathcal{U}_{\infty}=\lim _{n \in \mathcal{N}} \mathcal{U}_{n}
$$

Example 4.B: The one-dimensional box. For each $j \in \mathbb{N}$ and $x \in\left[-\frac{1}{2}, \frac{1}{2}\right]$, we write 


$$
\beta_{j, \infty}(x)= \begin{cases}\sqrt{2} \cos (\pi(j+1) x) & \text { if } j \text { is even, } \\ \sqrt{2} \sin (\pi(j+1) x) & \text { if } j \text { is odd. }\end{cases}
$$

Let $\mathcal{L}_{\infty}$ be the Hilbert space with orthonormal basis $\left\{\beta_{j, \infty}: j \in \mathbb{N}\right\}$. Let $\mathcal{S}$ be the dense subspace of $\mathcal{L}_{\infty}$ consisting of the smooth functions $\left[-\frac{1}{2}, \frac{1}{2}\right] \rightarrow \mathrm{C}$. The box, in one dimension, is the conservative system $\mathcal{U}_{\infty}$ whose Hamiltonian $\hat{H}_{\infty}$ has domain $\mathcal{S}$ and is given by

$$
\hat{H}_{\infty} \phi(x)=-d^{2} \phi(x) / d x^{2}
$$

for $\phi \in \mathcal{S}$. Evidently $\hat{H}_{\infty} \beta_{j, \infty}=\lambda_{j, \infty} \beta_{j, \infty}$ where $\lambda_{j, \infty}=\pi^{2}(j+1)^{2}$.

Again, let $\mathcal{N}$ be the set of positive odd integers. Let $\mathcal{L}_{n}$ be the $n$-dimensional inner product space consisting of the complex-valued functions on the integers $X$ lying in the interval $-n / 2$ $<X<n / 2$. As in the previous example, we replace the differential operator $-d^{2} / d x^{2}$ with a difference operator $\hat{H}_{n}$, but this time the sample-points indexed by $(1-n) / 2$ and $(n-1) / 2$ are to be interpreted as end-points (they are no longer interpreted as being adjacent). Writing $n=2 l$ +1 , we put

$$
\hat{H}_{n} \psi(X)=\left\{\begin{array}{l}
n^{2}(2 \psi(-l)-\psi(1-l)) \quad \text { if } X=-l, \\
n^{2}(-\psi(X-1)+2 \psi(X)-\psi(X+1)) \text { if }-l<X<l, \\
n^{2}(-\psi(l-1)+2 \psi(l)) \quad \text { if } X=l .
\end{array}\right.
$$

The operator $\hat{H}_{n}$ is diagonalized by the orthonormal basis $\mathcal{B}_{n}=\left\{\beta_{j, n}: 0 \leqslant j \leqslant n-1\right\}$ of $\mathcal{L}_{n}$, where

$$
\beta_{j, n}(X)= \begin{cases}\sqrt{\frac{2}{n-1}} \cos \left(\frac{\pi(j+1) X}{n+1}\right) & \text { if } j \text { is even, } \\ \sqrt{\frac{2}{n+1}} \sin \left(\frac{\pi(j+1) X}{n+1}\right) & \text { if } j \text { is odd. }\end{cases}
$$

In fact, $\hat{H}_{n} \beta_{j, n}=\lambda_{j, n} \beta_{j, n}$ where $\lambda_{j, n}=2(1-\cos (\pi(j+1) /(n+1)))$.

We realize $\left(\mathcal{L}_{n}\right)_{n}$ as an inductive resolution of $\mathcal{L}_{\infty}$ by defining $\operatorname{res}_{n}: \mathcal{S} \rightarrow \mathcal{L}_{n}$ by the same formula as in Example 4.A. A straightforward calculation yields, for all $j \in \mathbb{N}$, all $x \in\left[-\frac{1}{2}, \frac{1}{2}\right]$ and all sequences $\left(X_{n}\right)_{n}$ of integers such that $x=\lim _{n \in \mathcal{N}} X_{n} / \sqrt{n}$, the point-wise convergence condition

$$
\beta_{j, \infty}(x)=\lim _{n \in \mathcal{N}} \sqrt{n} \beta_{j, n}\left(X_{n} / n\right) .
$$

The norms $\left\|\beta_{j, n}\right\|$ are all unity, and, in particular, they are bounded. In Ref. 7, Theorem 3.1, it was proved that point-wise convergence of vectors with bounded norms implies convergence; in particular,

$$
\beta_{j, \infty}=\lim _{n \in \mathcal{N}} \beta_{j, n} .
$$

Observing that each $\lambda_{j, \infty}=\lim _{n \in \mathcal{N}} \lambda_{j, n}$, we again conclude from Proposition 3.7 that

$$
\mathcal{U}_{\infty}=\lim _{n \in \mathcal{N}} \mathcal{U}_{n}
$$

Example 4.C: The Harper function harmonic oscillator. In this example and the next, we review some results from Refs. 11 and 12, and we show how that material can be streamlined using Proposition 3.7. Recall that $L^{2}(\mathbb{R})$ has an orthonormal basis $\left\{h_{j, \infty}: j \in \mathbb{N}\right\}$ consisting of the functions $h_{j, \infty}: \mathrm{R} \rightarrow \mathrm{C}$, called the Hermite-Gaussians, which are given by 


$$
h_{j, \infty}(x)=C_{j} e^{-x^{2} / 2} H_{j}(x),
$$

where $H_{j}$ is the Hermite polynomial of degree $j$, and $C_{j}$ is a positive real normalization constant. The continuum harmonic oscillator is defined to be the conservative system $\mathcal{U}_{\infty}=\left\{\hat{U}_{\infty}(t): t\right.$ $\in \mathbb{R}\}$ whose Hamiltonian $\hat{H}_{\infty}$ is given by $\hat{H}_{\infty} \psi(x)=\left(-d^{2} / d x^{2}+x^{2}\right) \psi(x)$, or equivalently, $\hat{H}_{\infty} h_{j, \infty}=(2 k+1) h_{j, \infty}$. Thus

$$
\hat{U}_{\infty}(t) h_{j, \infty}=e^{-(2 j+1) i t} h_{j, \infty} .
$$

Let $\mathcal{N}$ be an infinite set of positive integers such that $\sqrt{n_{2} / n_{1}} \in \mathbb{Z}$ for all $n_{1}, n_{2} \in \mathcal{N}$ with $n_{1}$ $\leqslant n_{2}$. (At one point in the discussion, we shall make use of this peculiar hypothesis on $\mathcal{N}$, but the assertions probably hold for any infinite set $\mathcal{N}$ of positive integers.) Given an element $n \in \mathcal{N}$, let $\mathcal{L}_{n}$ be the $n$-dimensional inner product space consisting of the functions $\mathrm{Z} \rightarrow \mathrm{C}$ with period $n$. We realize $\left(\mathcal{L}_{n}\right)_{n}$ as an inductive resolution of $L^{2}(\mathbb{R})$ by defining restriction maps $\operatorname{res}_{n}: \mathcal{S}(\mathbb{R}) \rightarrow \mathcal{L}_{n}$ such that

$$
\operatorname{res}_{n}(\phi)(X)=(n / 2 \pi)^{-1 / 4} \phi\left((n / 2 \pi)^{-1 / 2} X\right)
$$

for $\phi \in \mathcal{S}(\mathbb{R})$ and $X \in \mathbb{Z}$. After Harper, ${ }^{13}$ Namias, ${ }^{1}$ Pei-Yeh ${ }^{3}$ and others, we define the Harper function harmonic oscillator to be the conservative system $\mathcal{U}_{n}$ on $\mathcal{L}_{n}$ with Hamiltonian $\hat{H}_{n}$ such that

$$
\hat{H}_{n} \psi(X)=\frac{n}{2 \pi}(-\psi(X-1)+(4-2 \cos (2 \pi i X / n)) \psi(X)-\psi(X+1))
$$

for $\psi \in \mathcal{L}_{n}$ and $X \in \mathbb{Z}$. The definition and enumeration of the Harper functions $\beta_{0, n}, \beta_{1, n}, \ldots$ may be found in Ref. 3; see also Refs. 11 and 12. The Harper functions comprise an orthonormal basis for $\mathcal{L}_{n}$, they are eigenvectors of $\hat{H}_{n}$, and by Ref. 12, Theorem 2.5,

$$
h_{j, \infty}=\lim _{n \in \mathcal{N}} \beta_{j, n}
$$

for all $j \in \mathcal{N}$. (It is here that the peculiar hypothesis on $\mathcal{N}$ is used.) Combining this result with Ref. 12, Lemmas 3.1 and 3.9, it is easy to show that the eigenvalue $\lambda_{j, n}$ of $\hat{H}_{n}$ associated with $\beta_{j, n}$ satisfies

$$
2 j+1=\lim _{n \in \mathcal{N}} \lambda_{j, n}
$$

Proposition 3.7 now yields

$$
\mathcal{U}_{\infty}=\lim _{n \in \mathcal{N}} \mathcal{U}_{n}
$$

As suggested in Ref. 7, Sec. 3, the peculiar hypothesis on $\mathcal{N}$ can perhaps be relaxed using results that were not available when Ref. 12 was written.

Example 4.D: The Harper function fractional Fourier transform. We continue to use the notation from Example 4.C. After Namias, the continuum FRFT is defined to be the conservative system $\mathcal{F}_{\infty}=\left\{\hat{F}_{\infty}^{t}: t \in \mathbb{R}\right\}$ such that

$$
\hat{F}_{\infty}^{t} h_{j, \infty}=e^{2 \pi i j t} h_{j, \infty} .
$$

As Namias observed, the continuum FRFT and the continuum harmonic oscillator are related by the equality 


$$
\hat{U}_{\infty}(t)=e^{-i t} \hat{F}_{\infty}^{-t / \pi} .
$$

Note that $\hat{F}^{1 / 4}$ is the usual Fourier transform. The Harper function FRFT comes in two versions, the import version $\mathcal{I}_{n}=\left\{\hat{I}_{n}^{t}: t \in \mathbb{R}\right\}$ and the domestic version $\mathcal{D}_{n}=\left\{\hat{D}_{n}^{t}: t \in \mathbb{R}\right\}$. The import version, defined by

$$
\hat{I}_{n}^{t} \beta_{j, n}=e^{2 \pi i j t} \beta_{j, n},
$$

is perhaps rather artificial (its eigenvalues being "imported" from the continuum FRFT), but it has the virtue that $\hat{F}_{n}^{1 / 4}$ is the usual discrete Fourier transform. The domestic version, defined by

$$
\hat{U}_{n}(t)=e^{-i t} \hat{D}_{n}^{-t / \pi},
$$

has the virtue that it has an explicit Hamiltonian, namely $\left(\hat{H}_{n}-1\right) / 2$. By Proposition 3.7,

$$
\mathcal{F}_{\infty}=\lim _{n \in \mathcal{N}} \mathcal{I}_{n}=\lim _{n \in \mathcal{N}} \mathcal{D}_{n}
$$

Example 4.E: The Kravchuk function harmonic oscillator. We retain the notation from Examples 4.C and 4.D, except that we now let $\mathcal{N}$ be any set of positive integers. Given $n \in \mathcal{N}$, let us write $n=2 l+1$, and let $\mathcal{X}_{n}$ be the set consisting of the $X$ such that $l+X$ and $l-X$ are both natural numbers. We write $L\left(\mathcal{X}_{n}\right)$ to denote the $n$-dimensional inner product space consisting of the complex-valued functions on $\mathcal{X}_{n}$. As in Ref. 7, Secs. 4 and 5, we realize $\left(L\left(\mathcal{X}_{n}\right)\right)_{n}$ as an inductive resolution of $L^{2}(\mathbb{R})$ by defining $\operatorname{res}_{n}: \mathcal{S}(\mathbb{R}) \rightarrow L\left(\mathcal{X}_{n}\right)$ such that

$$
\operatorname{res}_{n}(\phi)(X)=l^{-1 / 4} \phi\left(l^{-1 / 2} X\right)
$$

for $\phi \in \mathcal{S}(\mathrm{R})$ and $X \in \mathcal{X}_{n}$. Recall (or see Ref. 7, Sec. 5) that the Kravchuk functions $h_{j, n}$ comprise an orthonormal basis $\left\{h_{j, n}: 0 \leqslant j \leqslant n-1\right\}$ for $L\left(\mathcal{X}_{n}\right)$. The Kravchuk functions are discrete analog of the Hermite-Gaussians, and arise from a binomial weight function in place of a Gaussian weight function. By Ref. 7, Theorem 5.1,

$$
h_{j, \infty}=\lim _{n \in \mathcal{N}} h_{j, n}
$$

for all $j \in \mathbb{N}$. After Ref. 14, the Kravchuk function harmonic oscillator is defined to be the conservative system $\hat{K}_{n}=\left\{\hat{K}_{n}(t): t \in \mathbb{R}\right\}$ on $\mathcal{L}_{n}$ such that

$$
\hat{H}_{n}(t) h_{j, n}=e^{-(2 j+1) i t} h_{j, n} .
$$

By Proposition 3.7,

$$
\mathcal{U}_{\infty}=\lim _{n \in \mathcal{N}} \mathcal{K}_{n}
$$

Example 4.F: The Kravchuk function fractional Fourier transform. We retain the notation from the previous three examples, $\mathcal{N}$ being any infinite set of positive integers. After Ref. 2, the Kravchuk function FRFT is defined to be the conservative system $\mathcal{F}_{n}=\left\{\hat{F}_{n}^{t}: t \in \mathbb{R}\right\}$ such that

$$
\hat{F}_{n}^{t} h_{j, n}=e^{2 \pi i j t} h_{j, n} .
$$

Equivalently, $\mathcal{F}_{n}$ may be defined by

$$
\hat{K}_{n}(t)=e^{-i t} \hat{F}_{n}^{-t / \pi}
$$


By Proposition 3.7,

$$
\mathcal{F}_{\infty}=\lim _{n \in \mathcal{N}} \mathcal{F}_{n}
$$

Comment: Advantages of the Kravchuk function FRFT over the Harper function FRFT. In applications of the Harper function FRFT, one must select either the import version, whose eigenvalues are integer powers of $e^{2 \pi i t}$, but whose Hamiltonian is not known explicitly, or else one must select the domestic version, whose Hamiltonian is $\left(\hat{H}_{n}-1\right) / 2$, but whose eigenvalues are not known explicitly. Either way, the eigenvectors - the Harper functions-lack a known explicit formula, and have to be calculated numerically. The eigenvectors of the Kravchuk function FRFT $\mathcal{F}_{n}$ are integer powers of $e^{2 \pi i t}$. As can be gleaned from Refs. 2 and 5, the Hamiltonian for $\mathcal{F}_{n}$ has a very simple description in terms of the $n$-dimensional irreducible representation of the Lie algebra su(2) (see also Ref. 4). The eigenvectors of $\mathcal{F}_{n}$ - the Kravchuk functions-are given by a complicated but explicit formula.

\section{SOME QUESTIONS AND REMARKS ON CONVERGENCE OF SPECTRA}

An alternative description of a conservative system is provided by the spectral measure associated with the Hamiltonian. Throughout this section, we consider conservative systems $\mathcal{U}_{\infty}$ $=\left\{\hat{U}_{\infty}(t): t \in \mathbb{R}\right\}$ on $\mathcal{L}_{\infty}$ and $\mathcal{U}_{n}=\left\{\hat{U}_{n}(t): t \in \mathbb{R}\right\}$ on each $\mathcal{L}_{n}$. Let $\hat{H}_{\infty}$ and $\hat{H}_{n}$ be the Hamiltonians for $\mathcal{U}_{\infty}$ and $\hat{U}_{n}$, respectively. If $\mathcal{U}_{\infty}=\lim _{n \in \mathcal{N}} \mathcal{U}_{n}$, how is the spectral measure for the Hermitian operator $\mathcal{H}_{\infty}$ related to the spectral measure for the operators $\mathcal{H}_{n}$ ? Or, more simply, how is the spectrum $\sigma\left(\hat{H}_{\infty}\right)$ (or the essential or residual spectrum) related to the spectra $\sigma\left(\hat{H}_{n}\right)$ ?

On the one hand, it would be desirable to have techniques for investigating the spectrum (or spectral measure) of an infinite-dimensional system by examining limiting properties of the spectra of finite-dimensional approximations. On the other hand, finite-dimensional systems are themselves of interest. (As a vague principle, any closed system of finite extent in space can have only finitely many independent nondecaying states.) Finite-dimensional systems are not always more amenable than infinite-dimensional systems (difference equations often have richer solutions than their analogous differential equations.) In connection with example 4.E, it is worth remembering that De Moivre, having established the correspondence between the Gaussian distribution and the binomial distribution, then employed the Gaussian as an approximation to the binomial. Continuum approximation to discrete phenomena has pervaded statistical techniques ever since. It is to be expected that results relating $\sigma\left(\hat{H}_{\infty}\right)$ and $\sigma\left(\hat{H}_{n}\right)$ could be usefully applied in either direction.

As regards practical methods for relating the spectra of discrete and continuum systems, the results in this article are simply not in competition with those in Ref. 8. We do not know whether or not their results can be extended to our more general context. (It should be mentioned that the examples considered in Sec. 4 are all, essentially, in the situation they considered.) The following result indictates that the questions above do have answers, and that our approach can be developed to yield alternative and more general methods.

Proposition 5.1: Suppose that $\mathcal{U}_{\infty}=\lim _{n} \mathcal{U}_{n}$. Suppose also that $\hat{H}_{\infty}$ and each $\hat{H}_{n}$ are bounded, and that the norms $\left\|\hat{H}_{n}\right\|$ are bounded. Then every point $\lambda \in \sigma\left(\hat{H}_{n}\right)$ is the limit $\lambda=\lim _{n} \lambda_{n}$ of points $\lambda_{n} \in \sigma\left(\hat{H}_{n}\right)$.

Proof: The condition $\lambda \in \sigma\left(\hat{H}_{\infty}\right)$ is equivalent to the condition that there exists a sequence $\left(\phi_{m}\right)_{m}$ of vectors in $\mathcal{L}_{\infty}$ such that $\left\|\phi_{m}\right\|=1$ and $\left\|\left(\hat{H}_{\infty}-\lambda\right) \phi_{m}\right\| \rightarrow 0$ as $m \rightarrow \infty$ (see, for instance, Ref. 15, Theorem 5.10). Since $\mathcal{S}$ is dense in $\mathcal{L}_{\infty}$, we may insist that each $\phi_{m} \in \mathcal{S}$. Let $\epsilon>0$, and fix $m$ such that $\left\|\left(\hat{H}_{\infty}-\lambda\right) \phi_{m}\right\| \leqslant \epsilon / 2$. By Proposition 3.3, the convergence hypothesis on $\mathcal{U}_{\infty}$ is equivalent to the condition that $\hat{H}_{\infty}=\lim _{n} \hat{H}_{n}$. Noting that $\lim _{n}\left\|\operatorname{res}_{n}\left(\phi_{m}\right)\right\|=1$, and putting $\psi_{n}$ $=\operatorname{res}_{n}\left(\phi_{m}\right) /\left\|\operatorname{res}_{n}\left(\phi_{m}\right)\right\|$, we have $\left\|\left(\hat{H}_{n}-\lambda\right) \psi_{n}\right\| \leqslant \epsilon$ for sufficiently large $n$. By a well-known criterion for existence of spectral points in an interval (see Ref. 12, Theorem 5.9), $\sigma\left(\hat{H}_{n}\right) \cap[\lambda$ $-\epsilon, \lambda+\epsilon] \neq \varnothing$. 
Corollary 5.2: In the situation of Proposition 5.1, suppose that the limits $\lim _{n} \mu_{n}$ of points $\mu_{n} \in \sigma\left(\hat{H}_{n}\right)$ comprise a discrete subset of $\mathbb{R}$. Then $\hat{H}_{n}$ is diagonalizable.

It seems probable that the boundedness condition in Proposition 5.1 can be removed by using a refinement of the argument (and the rider to Stone's theorem as recorded in Ref. 9, Theorem 13.1). A more systematic option would be to wait for that to become a corollary of a result expressing the condition $\mathcal{U}_{\infty}=\lim _{n} \mathcal{U}_{n}$ in terms of the spectral measures. We end with a few comments in this direction. Consider an interval $I$ in $\mathbb{R}$. Write $\bar{I}$ and $I^{\circ}$ for the closure and the interior. Let $E_{I, \infty}$ and $E_{I, n}$ be the corresponding projections to $\mathcal{L}_{\infty}$ and $\mathcal{L}_{n}$ associated with $\hat{H}_{\infty}$ and $\mathcal{H}_{n}$. To see that convergence of the sequence $\left(\hat{H}_{n}\right)_{n}$ does not imply convergence of the sequence $\left(E_{I, n}\right)_{n}$, let $a$ be an end-point of $I$, and let $\hat{H}_{n}=\left(a+(-2)^{n}\right) \hat{1}$.

Question 5.A: Are the following conditions equivalent?

(1) $\mathcal{U}_{\infty}=\lim _{n} \mathcal{U}_{n}$.

(2) If $\psi_{\infty}=\lim _{n} \psi_{n}$ with $\psi_{n} \in E_{I, n} \mathcal{L}_{n}$, then $\psi_{\infty} \in E_{I, n}^{-} \mathcal{L}_{\infty}$.

(3) If $\psi_{\infty}=\lim _{n} \psi_{n}$ with $\psi_{\infty} \in E_{I^{\circ}, n} \mathcal{L}_{\infty}$ and $\left\|\psi_{\infty}\right\|=\lim _{n}\left\|\psi_{n}\right\|$, then $\lim _{n}\left\|\left(\hat{1}-\hat{E}_{I, n}\right) \psi_{n}\right\|=0$.

${ }^{1}$ V. Namias, "The fractional order Fourier transform and its applications to quantum mechanics," J. Inst. Math. Appl. 25, 214-265 (1980).

${ }^{2}$ N. M. Atakishiyev and K. B. Wolf, "Fractional Fourier-Kravchuk transform," J. Opt. Soc. Am. 14, 1467-1477 (1997).

${ }^{3}$ S-C. Pei and M-H. Yeh, "Improved discrete fractional Fourier transform," Opt. Lett. 22, 1047-1049 (1997).

${ }^{4}$ L. Barker, "Continuum quantum systems as limits of discrete quantum systems, IV: affine canonical transforms" (preprint).

${ }^{5}$ N. M. Atakishiyev, S. M. Chumakov, and K. B. Wolf, "Wigner distribution for finite systems," J. Math. Phys. 39, 6247-6269 (1998)

${ }^{6}$ L. Barker, "Continuum quantum systems as limits of discrete quantum systems, I: state vectors," J. Funct. Anal. (to appear).

${ }^{7}$ L. Barker, "Continuum quantum systems as limits of discrete quantum systems, II: state functions," J. Phys. A 22, 4673-4682 (2001).

${ }^{8}$ T. Digernes, V. S. Varadarájan, and S. R. S. Varadhan, "Finite approximations to quantum systems," Rev. Mod. Phys. 6, 621-648 (1994).

${ }^{9}$ K. R. Parthasarathy, An Introduction to Quantum Stochastic Calculus (Birkhäuser, Basel, 1992).

${ }^{10}$ J. Weidmann, Linear Operators in Hilbert Spaces (Teubner, Stuttgart, 1976; English edition by Springer, Berlin, 1980).

${ }^{11}$ L. Barker, Ç. Candan, T. Hakioğlu, A. Kutay, and H. M. Ozaktas, "The discrete harmonic oscillator, Harper's equation, and the discrete fractional Fourier transform," J. Phys. A 33, 2209-2222 (2000).

${ }^{12}$ L. Barker, "The discrete fractional Fourier transform and Harper's equation," Mathematika (to appear).

${ }^{13}$ P. G. Harper, "Single band motion of conduction electrons in a uniform magnetic field," Proc. Phys. Soc. 68, 874-878 (1955).

${ }^{14}$ N. M. Atakishiev and S. K. Suslov, "Difference analogues of the harmonic oscillator (English translation)," Teor. Mat. Fiz. 85, 64-73 (1990).

${ }^{15}$ P. D. Hislop and I. M. Sigal, Introduction to Spectral Theory, with Applications to Schrödinger Operators (Springer, Berlin, 1996) 